\title{
Idiopathic Seidlmayer's Purpura: A Case Report
}

\author{
Maria Cristina Maggio Fabrizia Ferraro Saveria Sabrina Ragusa \\ Umberto Corpora Giovanni Corsello \\ Department Pro.S.A.M.I., Ospedale dei Bambini 'G. Di Cristina', ARNAS Palermo, Palermo, \\ Italy
}

\section{Key Words}

Acute hemorrhagic edema of infancy $\cdot$ Seidlmayer's purpura $\cdot$ Vasculitis $\cdot$ Corticosteroids

\begin{abstract}
Acute hemorrhagic edema of infancy (AHEI) was considered a rare form of Henoch-Schönlein purpura; however, it is now regarded as an independent disease typically involving patients aged 4-24 months. The authors describe the clinical case of a toddler aged 8 months, with skin erythematous pomphoid lesions, treated at home with topical steroids without benefits. The appearance of new lesions and the worsening of the previous skin signs induced the parents to drive the child to the hospital. The medical history revealed the administration of a vaccine dose 2 months before.

(c) 2014 S. Karger AG, Basel
\end{abstract}

\section{Introduction}

About 100 cases of acute hemorrhagic edema of infancy (AHEI) have been published in the medical literature worldwide [1-4]. Although initially regarded as a variant of HenochSchönlein purpura, it is now considered a separate entity: in fact, it shows infrequently visceral involvement and IgA skin depositions [5]. Furthermore, these patients tend to have a better prognosis than Henoch-Schönlein purpura patients. The onset age for AHEI usually ranges between 4 and 24 months, but it spreads from birth to 60 months. AHEI, also defined as Seidlmayer's purpura, is characterized by the triad: fever, edema and purpura. The latter is usually rosette-, annular-, or targetoid-shaped, and is primarily observed over the face, ears and extremities in a nontoxic infant [5]. The development and the rapidity of the onset of the skin lesions are typical and more frequent in winter. Skin lesions are dramatic both in appearance and rapidity of onset. However, AHEI is a self-limited short-duration disease, 
usually lasting less than 3 weeks. Long-term sequelae are unlikely and relapses are uncommon [4].

\section{Case Report}

The authors describe the clinical case of an 8-month-old toddler, in good health, who presented to our division due to skin erythematous pomphoid lesions, treated at home with topical steroids without benefits. The appearance of new lesions and the worsening of the previous ones induced the parents to drive the child to the hospital.

At the time of admission the patient was apyretic. The purpuric lesions were localized on the face (fig. 1, fig. 2), on the distal ends of the upper and lower limbs (fig. 3) and on the gluteal region. Leukocytes, C-reactive protein, transaminases, urine, blood urea nitrogen and creatinine were in the normal ranges. Furthermore, the good clinical condition of the child allowed ruling out a septic framework. A scan of the abdomen excluded the presence of thickening of intestinal loops, suggestive of an involvement of the small vessel district. A dermatological evaluation confirmed the suspicion of AHEI.

Serological assays of IgM and IgG specific for Coxsackie, Epstein-Barr, Parvovirus, adenovirus, Mycoplasma pneumoniae and Chlamydia excluded a recent infection. Antinuclear antibodies and antineutrophil cytoplasmic antibodies were undetectable. A nasopharyngeal swab was negative for pathogenic bacteria and viruses such as influenza, parainfluenza, respiratory syncytial virus and group A $\beta$-hemolytic streptococcus.

The medical history revealed the administration of a vaccine dose 2 months before. The long period between the vaccine and the AHEI onset suggested excluding an etiopathogenetic link with the vaccination.

During the admission period the child presented stability of the clinical condition, except for dermatological lesions that showed a worsening clinical outcome. The histopathology of a vasculitis lesion was characterized by small-vessel fibrinoid necrosis of the wall. Furthermore, a perivascular neutrophilic infiltrate and dermal leukocytoclasia, describing leukocytoclastic vasculitis, were detected by histology. Direct immunofluorescence did not detect IgA deposits. The development of purulent conjunctivitis needed treatment with tobramycin drops.

The worsening of the skin lesions with a tendency to ulcerative evolution in the left ear and in one foot (fig. 2) suggested treatment with methylprednisolone $(1 \mathrm{mg} / \mathrm{kg} / \mathrm{day}$ with benefit, tapering on the 6th day and completely suspended after 8 days).

During the follow-up, the child was in excellent condition, and the skin lesions disappeared without new lesions. Fecal occult blood and urine examination were negative during the acute phase of the illness and the follow-up. The monitoring of urine for hematuria and proteinuria was recommended at 1, 3 and 6 months, and the child did not present any relapse of the disease during the follow-up period.

\section{Discussion and Conclusions}

Informed consent was obtained from the patient's parents. Usually no specific treatment is required for AHEI. The role of corticosteroids is limited to complicated forms.

In our patient, corticosteroids were necessary due to the severity of the dermatological lesions, with a successful improvement in the clinical evolution. Recognition of this rare purpura will avoid misdiagnosis, parental anxiety and inappropriate treatment. 
Maggio et al.: Idiopathic Seidlmayer's Purpura: A Case Report

\section{Disclosure Statement}

The authors report that they have no conflicts of interest.

\section{References}

1 Crowe MA, Jonas PP: Acute hemorrhagic edema of infancy. Cutis 1998;62:65-66.

-2 Karremann M, Jordan AJ, Bell N, Witsch M, Dürken M: Acute hemorrhagic edema of infancy: report of 4 cases and review of the current literature. Clin Pediatr (Phila) 2009;48:323-326.

-3 Michael DJ: Acute hemorrhagic edema of infancy. Dermatol Online J 2006;12:10.

-4 Sites LY, Woodmansee CS, Wilkin NK, Hanson JW, Skinner RB Jr, Shimek CM: Acute hemorrhagic edema of infancy: case reports and a review of the literature. Cutis 2008;82:320-324.

-5 Saraclar Y, Tinaztepe K, Adalioglu G, Tuncer A: Acute hemorrhagic edema of infancy (AHEI) - a variant of Henoch-Schönlein purpura or a distinct clinical entity? J Allergy Clin Immunol 1990;86:473-483.

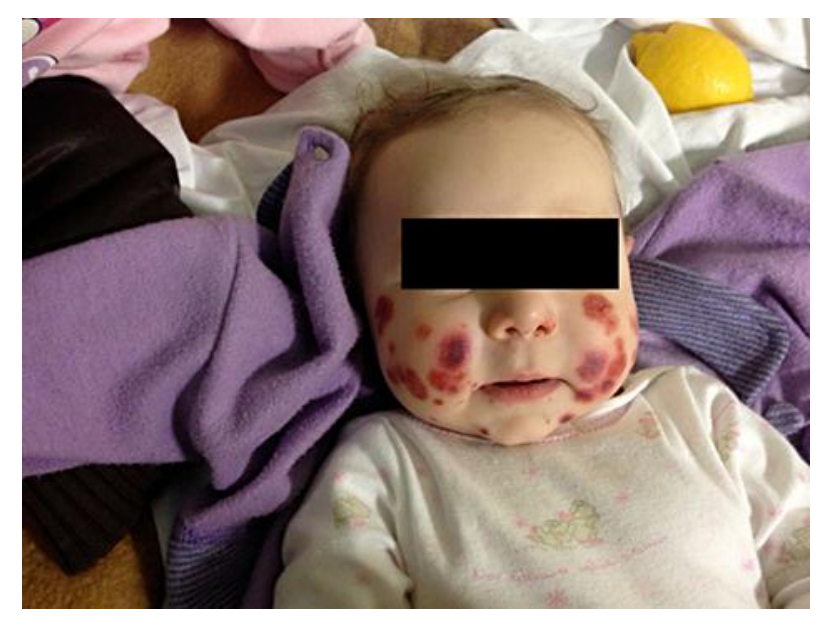

Fig. 1. Skin lesions of the face. 


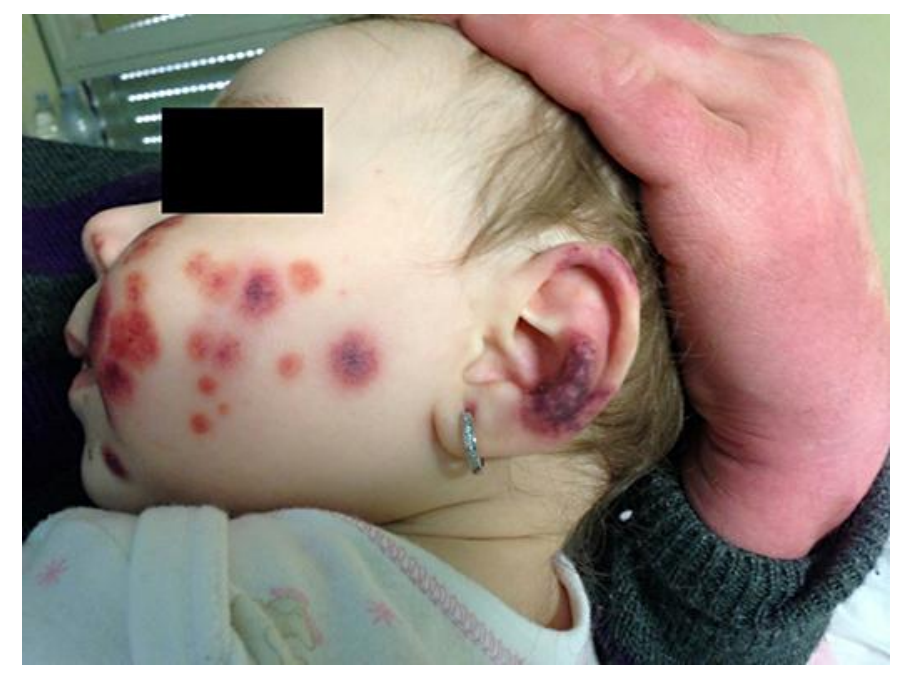

Fig. 2. Worsening of the skin lesions of the ear.

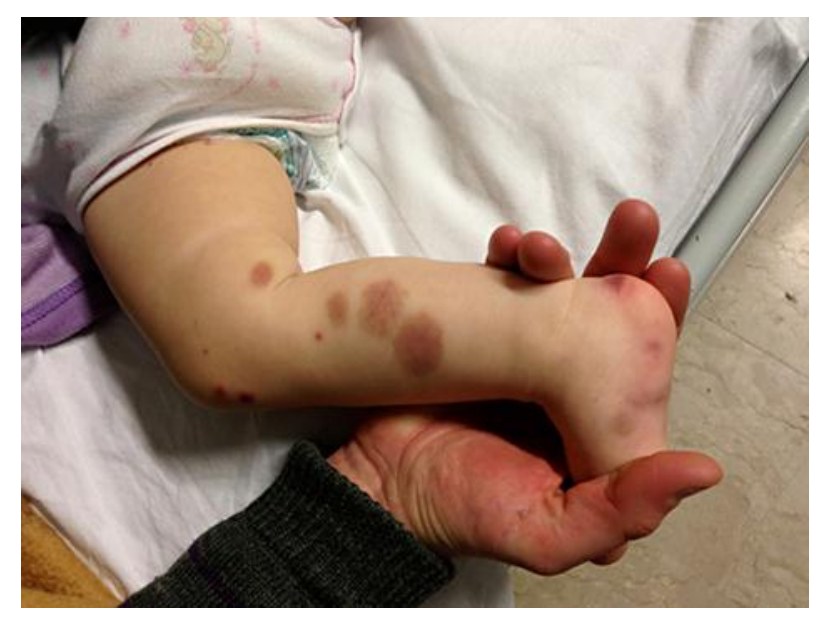

Fig. 3. Skin lesions of the leg. 